\title{
A Feedforward Inhibitory Circuit Mediated by CBI-Expressing Fast-Spiking Interneurons in the Nucleus Accumbens
}

\author{
William J Wright', Oliver M Schlüter' and Yan Dong*,I \\ 'Department of Neuroscience, University of Pittsburgh, Pittsburgh, PA, USA
}

The nucleus accumbens (NAc) gates motivated behaviors through the functional output of principle medium spiny neurons (MSNs), whereas dysfunctional output of NAc MSNs contributes to a variety of psychiatric disorders. Fast-spiking interneurons (FSIs) are sparsely distributed throughout the NAc, forming local feedforward inhibitory circuits. It remains elusive how FSI-based feedforward circuits regulate the output of NAc MSNs. Here, we investigated a distinct subpopulation of NAc FSls that express the cannabinoid receptor type-I (CBI). Using a combination of paired electrophysiological recordings and pharmacological approaches, we characterized and compared feedforward inhibition of NAc MSNs from $\mathrm{CBI}{ }^{+}$FSIs and lateral inhibition from recurrent MSN collaterals. We observed that $\mathrm{CBI}{ }^{+}$FSls exerted robust inhibitory control over a large percentage of nearby MSNs in contrast to local MSN collaterals that provided only sparse and weak inhibitory input to their neighboring MSNs. Furthermore, $\mathrm{CBI} I^{+}$FSI-mediated feedforward inhibition was preferentially suppressed by endocannabinoid (eCB) signaling, whereas MSN-mediated lateral inhibition was unaffected. Finally, we demonstrated that $\mathrm{CBI}{ }^{+} \mathrm{FSI}$ synapses onto MSNs are capable of undergoing experience-dependent long-term depression in a voltageand eCB-dependent manner. These findings demonstrated that $\mathrm{CB} \mathrm{I}^{+}$FSIs are a major source of local inhibitory control of MSNs and a critical component of the feedforward inhibitory circuits regulating the output of the NAc.

Neuropsychopharmacology (2017) 42, II 46-I I56; doi:I 0.1 038/npp.20 I 6.275; published online 4 January 2017

\section{INTRODUCTION}

The nucleus accumbens (NAc) has been conceptualized as a limbic-motor interface gating motivated behaviors, whereas deviated functional output of the NAc contributes to a variety of psychiatric states, including addiction and depression (Hyman et al, 2006; Mogenson et al, 1980; Nestler et al, 2002; Wise, 1987). The functional output of the NAc is mediated by medium spiny neurons (MSNs), whose activation is driven by the integration of excitatory inputs (Brog et al, 1993; Meredith et al, 1992; Wilson and Kawaguchi, 1996). An essential component regulating the activation and output of MSNs are local feedforward inhibitory circuits. In the dorsal striatum, the output of MSNs is grossly regulated by fast-spiking interneurons (FSIs) (Koos and Tepper, 1999; Tepper et al, 2008). Although greatly outnumbered by MSNs, FSIs are distributed throughout the ventral and dorsal striatum, with each FSI innervating hundreds of MSNs (Luk and Sadikot, 2001; Tepper et al, 2008). These FSIs provide tonic inhibition as well as timing-dependent

* Correspondence: Dr Y Dong, Department of Neuroscience, University of Pittsburgh, A2IO Langley Hall, 5th and Ruskin Avenue, Pittsburgh, PA 15260, USA, Tel: + I 412624 3140, Fax: + I 412624 9198, E-mail: yandong@pitt.edu

Received 25 September 2016; revised I 8 November 2016; accepted 3 December 2016; accepted article preview online 8 December 2016 feedforward inhibition to MSNs upon excitation (Koos and Tepper, 1999; Mallet et al, 2005). The function of FSIs has primarily been characterized in the dorsal striatum. Although sharing many features, the NAc also exhibits distinct circuitry differences with the dorsal striatum (Kupchik et al, 2015; Zhou et al, 2003). It remains elusive how FSI-mediated feedforward circuits function in the NAc to orchestrate the output of NAc MSNs.

We recently identified a subpopulation of neurons in the NAc that uniquely express the cannabinoid receptor type-1 (CB1) (Winters et al, 2012). Unlike the dorsal striatum, $\mathrm{CB} 1$-expressing $\left(\mathrm{CB}^{+}\right)$neurons in the NAc are exclusively FSIs, with indistinguishable biophysical properties from parvalbumin (PV)-expressing FSIs (Winters et al, 2012). Because endocannabinoids (eCBs) are released by activated MSNs, $\mathrm{CB}^{+}{ }^{+}$FSI-mediated feedforward inhibition is regulated by MSN activity. Our present study targeted this unique, yet underexplored, population of NAc FSIs, and demonstrated that $\mathrm{CB}^{+}$FSIs exerted robust inhibitory control over MSNs in contrast to local MSN collaterals that provided only sparse and weak inhibition to neighboring MSNs. Furthermore, the $\mathrm{CB}^{+}$FSI-mediated feedforward inhibition was also preferentially suppressed by $\mathrm{eCB}$ signaling that is capable of inducing long-term depression (LTD) at these synapses. These results suggest $\mathrm{CB} 1^{+}$FSIs as a unique source of local inhibitory control of MSNs and a critical component of the circuits governing the output of the NAc. 
a

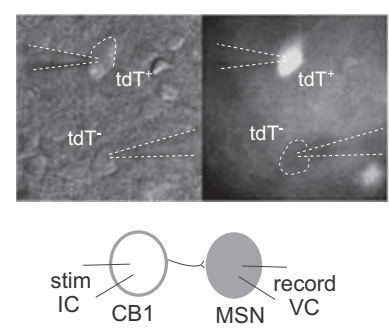

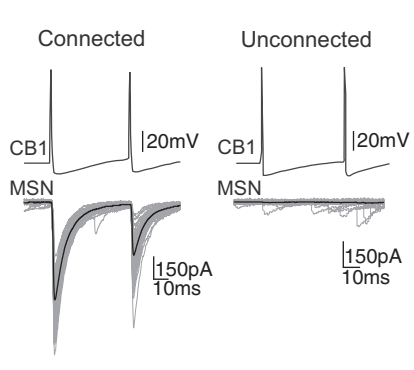

b

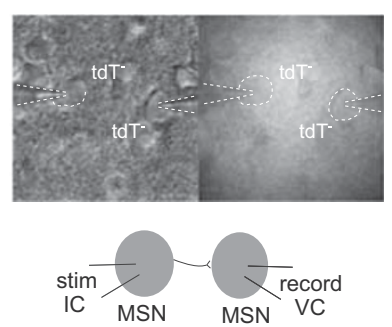

Connected

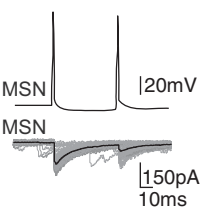

Unconnected

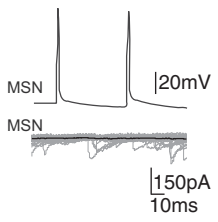

C

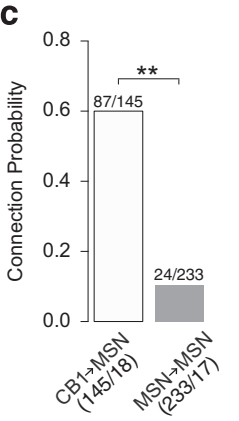

h

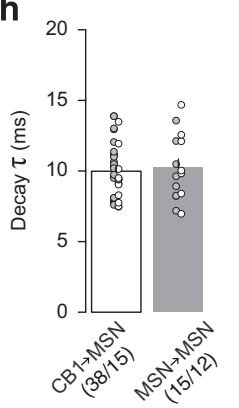

d $\quad \stackrel{\text { Male }}{\circ \text { Female }} \quad$ e

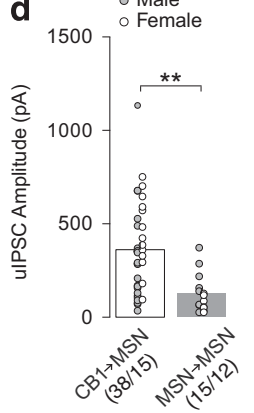

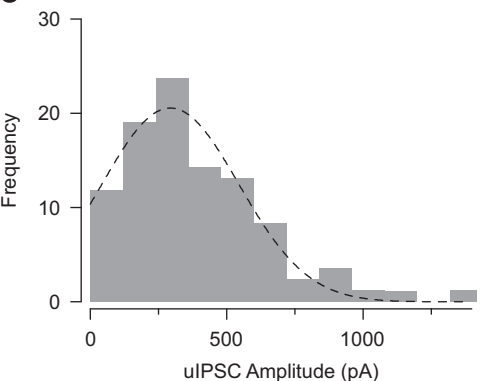

f

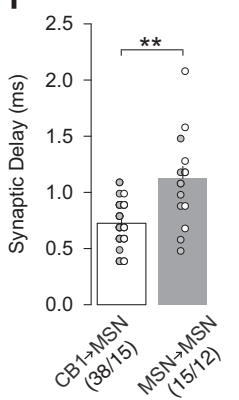

g

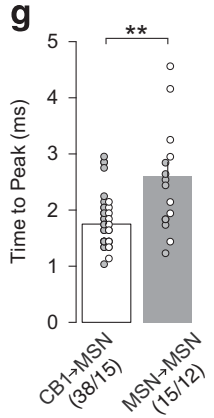

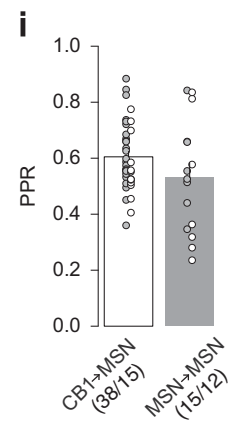

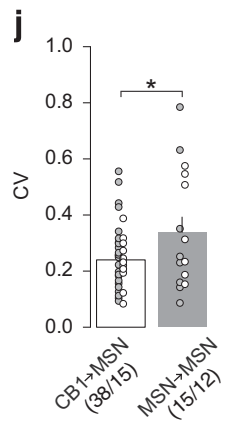

k
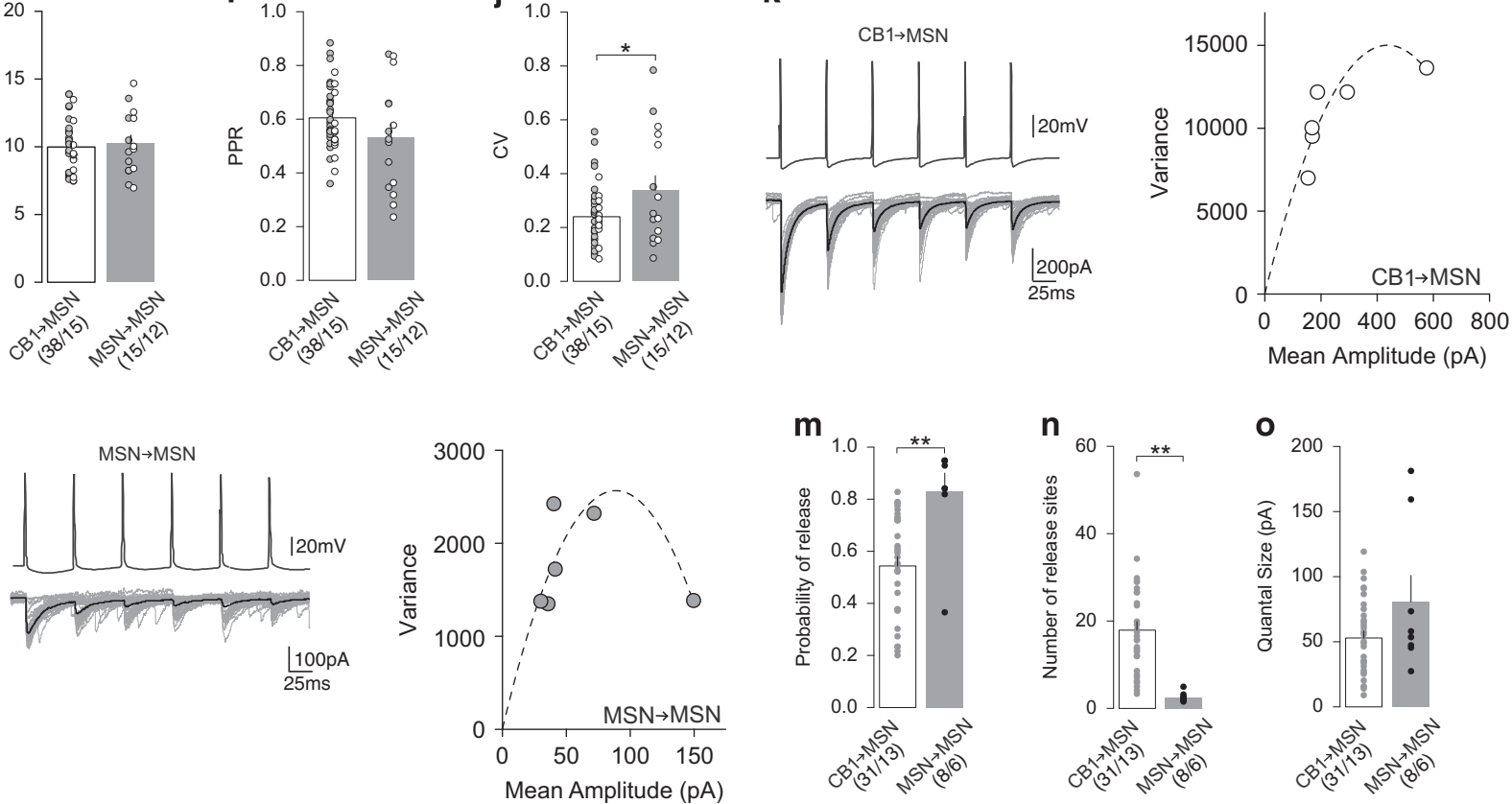
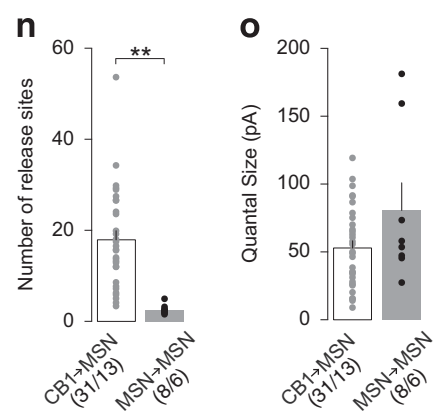

Figure I Comparison of CBI-to-MSN and MSN-to-MSN inhibitory synaptic transmissions. (a) Representative images and schematic diagram (left) of paired recordings between $\mathrm{CBI}^{+} \mathrm{FSI}\left(\mathrm{tdT}^{+}\right.$) and $\mathrm{MSN}\left(\mathrm{tdT}^{-}\right.$), and representative ulPSC traces from functionally connected (middle) and unconnected (right) $\mathrm{CBI}$-toMSN pairs. (b) Representative images and schematic diagram (left) of paired recordings between MSN (tdT ${ }^{-}$) and MSN (tdT ${ }^{-}$), and representative uIPSC traces from functionally connected (middle) and unconnected (right) MSN-to-MSN pairs. (c) Summary showing CBI-to-MSN pairs exhibited a greater probability of connectivity than MSN-to-MSN pairs. (d) Summary showing amplitude of ulPSCs was greater at CBI-to-MSN synapses than MSN-toMSN synapses. (e) Distribution of uIPSCs with a Gaussian fit showing a monomodal distribution. (f) Summary showing uIPSCs evoked at CBI-to-MSN synapses had a shorter synaptic delay than uIPSCs at MSN-to-MSN synapses. (g) Summary showing ulPSCs evoked at CBI-to-MSN synapses had shorter time to peak than uIPSCs MSN-to-MSN synapses. (h) Summary showing uIPSCs evoked at CBI-to-MSN and MSN-to-MSN synapses have similar decay kinetics. (i) Summary showing similar PPR of uIPSC responses at CBI-to-MSN and MSN-to-MSN synapses. (j) Summary showing uIPSC responses at MSN-to-MSN synapses had a greater CV than uIPSC responses at CBI-to-MSN synapses. (k) Example of uIPSC traces (left) and their hyperbolic variance mean fitted plot (right) at CBI-to-MSN synapses upon 6-pulse $20 \mathrm{~Hz}$ stimulation. (I) Example of ulPSC traces (left) and their hyperbolic variance mean fitted plot (right) at MSN-to-MSN synapses upon 6-pulse $20 \mathrm{~Hz}$ stimulation. (m) Summary showing MSN-to-MSN synapses had a higher presynaptic release probability than CBI-to-MSN synapses. (n) Summary showing CBI-to-MSN pairs had a greater number of release sites than MSN-to-MSN pairs. (o) Summary showing similar quantal size at $\mathrm{CBI}$-to-MSN and MSN-to-MSN synapses. $\mathrm{n} / \mathrm{m}$ represents number of cells/number of animals. $* P<0.05$, *** $<0.01$. Error bars represent SEM. 


\section{MATERIALS AND METHODS}

\section{Animals}

CB1-tdTomato knock-in mice ( $25 \mathrm{~g} ; 50-90$ days old) (Winters et al, 2012) were used in all experiments. We examined potential sex differences for the basic functional properties of $\mathrm{CB}^{+}$FSI-to-MSN and MSN-to-MSN synapses and found no differences between sexes (CB1-to-MSN or MSN-to-MSN, $p>0.05$ for all measures, $t$-test; Figure 1 ). Therefore, for all subsequent experiments, data from both sexes were combined for final data analysis.

\section{Electrophysiology Recordings}

All recordings were made in the medial NAc shell. Paired recordings were used to assess the properties of unitary inhibitory postsynaptic currents (uIPSCs) from $\mathrm{CB}^{+}{ }^{+}$FSI-toMSN synapses and synapses between MSNs. To record connected pairs, postsynaptic MSNs were randomly sampled within a $\sim 100 \mu \mathrm{m}$ radius from the presynaptic cell, within the approximate axonal arbor of FSIs and MSNs ( 200$300 \mu \mathrm{m})$ (Kawaguchi, 1993; Koos et al, 2004). The presynaptic patch pipette was filled with potassium-based internal solution (in mM: $130 \mathrm{~K}$ methanesulfate, $10 \mathrm{KCl}, 10$ Hepes, 0.4 EGTA, $2 \mathrm{MgCl}_{2}$, 3 Mg-ATP, $0.25 \mathrm{Na}$-GTP; pH 7.3, $290 \mathrm{mM} \mathrm{mOsm}$ ), whereas postsynaptic pipettes were filled with high-chloride, cesium-based internal solution (in mM: $15 \mathrm{CsMeSO}^{4}, 140 \mathrm{CsCl}, 4 \mathrm{TEA}-\mathrm{Cl}$, 0.4 EGTA (Cs), 20 Hepes, 3 Mg-ATP, 0.25 Na-GTP, 5 QX-314(Br), pH 7.3, $290 \mathrm{mM} \mathrm{mOsm})$ to enhance IPSCs. This arrangement allowed for connectivity to be tested in one direction. Simultaneous dual voltage-clamp recordings were used to compare excitatory postsynaptic currents (EPSCs) in $\mathrm{CB}^{+}$ FSIs and MSNs. Cells were held at $-70 \mathrm{mV}$ unless otherwise stated. For all EPSC recordings, electrodes were filled with cesium-based internal solution (in mM: $135 \mathrm{CsMeSO}_{4}, 5$ CsCl, 5 TEA-Cl, 0.4 EGTA (Cs), 20 Hepes, 3 Mg-ATP, 0.25 Na-GTP, 1 QX-314(Br), pH 7.3, 290 mM mOsm). Picrotoxin $(100 \mu \mathrm{M})$ was included in aCSF to inhibit $\mathrm{GABA}_{\mathrm{A}}$-mediated currents. All chemicals used were purchased from SigmaAldrich (St Louis, MO) or Tocris (UK).

\section{Data Acquisition, Analysis, and Statistics}

Results are shown as mean \pm SEM. Statistical significance was assessed with Fisher's exact test, paired or unpaired twotailed $t$-tests, one-way ANOVA, or two-way ANOVA with repeated measures, as specified. Cell-based statistics were performed for electrophysiology data. Significance was set at $\alpha=0.05$. Sample size for electrophysiology experiments are presented as $n / m$, where " $n$ " refers to the number of cells and " $\mathrm{m}$ " to the number of animals examined.

Detailed experimental procedures are provided in the Supplementary Materials.

\section{RESULTS}

\section{CB1 \pm FSIs Provide More Robust Inhibition to MSNs than Recurrent MSN Collaterals}

In addition to FSI-mediated feedforward inhibition, MSNs also receive lateral inhibition from recurrent MSN collaterals
(Tepper et al, 2008). Therefore, we compared inhibitory inputs to MSNs from $\mathrm{CB}^{+}$FSIs $v s$ MSN collaterals to determine the relative weight of inhibition of these two inhibitory circuits. To isolate synaptic transmission between these distinct circuits, we performed paired recordings between either $\mathrm{CB1}^{+}$FSIs and MSNs (CB1-to-MSN) or between neighboring MSNs (MSN-to-MSN) to evoke uIPSCs in a mouse line in which $\mathrm{CB}^{+}$neurons are genetically labeled with tdTomato (CB1-tdT mice) (Figure 1a and b).

We observed $\sim 60.0 \%$ (87/145) of CB1-to-MSN pairs recorded were functionally connected in contrast to $\sim 10.3 \% \quad(24 / 233)$ found between MSN-to-MSN pairs (Fisher's exact test; $p=0.00$; Figure 1c). These different connectivity rates suggest that $\mathrm{CB1}^{+}$FSIs exerts more global influence over the activity of local MSNs than neighboring MSNs. For connected pairs, the amplitude of uIPSCs was $\sim 2.8$-fold greater at CB1-to-MSN synapses $(361.60 \pm 38.15 \mathrm{pA})$ than MSN-to-MSN synapses (128.20 $\pm 26.01 \mathrm{pA}$ ) (unpaired two-tailed $t$-test: $t_{51}=3.69, p=0.00$; Figure 1d), further demonstrating that $\mathrm{CB}^{+}$FSIs provide stronger inhibitory input to MSNs than MSNs. Our previous results suggest that $\mathrm{CB1}^{+}$FSIs can be divided into two subpopulations, $\mathrm{PV}^{+}$and $\mathrm{PV}^{-}$FSIs (Winters et al, 2012). A distribution analysis indicates a monomodal pseudonormal distribution of CB1-to-MSN uIPSCs, implying a similar synaptic connectivity of these two subpopulations of $\mathrm{CB}^{+}$ FSIs to MSNs (Figure 1e). Our further analyses indicate that CB1-to-MSN uIPSCs displayed a shorter synaptic delay (CB1-to-MSN, $0.73 \pm 0.03 \mathrm{~ms}$; MSN-to-MSN, $1.13 \pm 0.11 \mathrm{~ms}$; unpaired two-tailed $t$-test: $t_{51}=4.86, p=0.00$; Figure 1f) and faster time to peak (CB1-to-MSN, $1.75 \pm 0.08 \mathrm{~ms}$; MSN-toMSN, $2.61 \pm 0.25 \mathrm{~ms}$; unpaired two-tailed $t$-test: $t_{51}=4.37$, $p=0.00$; Figure $1 \mathrm{~g}$ ) than MSN-to-MSN uIPSCs, whereas there was no difference in the decay kinetics (CB1-to-MSN, $\tau=9.99 \pm 0.31 \mathrm{~ms} ; \quad M S N-$ to-MSN, $\tau=10.27 \pm 0.60 \mathrm{~ms}$; unpaired two-tailed $t$-test: $t_{51}=0.44, p=0.66$; Figure $1 \mathrm{~h}$ ). Faster activation kinetics suggests that synapses from $\mathrm{CB} 1^{+}$FSIs may be located on more proximal somatodendritic sites of MSNs than synapses from MSN collaterals, and therefore more effective in transmitting synaptic signals.

Further analyses indicate that CB1-to-MSN and MSN-toMSN synapses exhibit different synaptic properties. We first measured the PPR and CV, differences in which may reflect differences in presynaptic release probability. No difference was detected in the PPR between CB1-to-MSN pairs $(0.61 \pm 0.02)$ and $M S N$-to-MSN pairs $(0.53 \pm 0.05)$ (unpaired two-tailed $t$-test: $t_{51}=1.64, p=0.11$; Figure 1i). In contrast, the $\mathrm{CV}$ of MSN-to-MSN pairs $(0.34 \pm 0.05)$ was significantly greater than CB1-to-MSN pairs $(0.24 \pm 0.02)$ (unpaired twotailed $t$-test: $t_{51}=2.22, p=0.03$; Figure $1 \mathrm{j}$ ). Similar PPRs suggest CB1-to-MSN and MSN-to-MSN synapses have similar presynaptic release probabilities, whereas a greater $\mathrm{CV}$ suggests that MSN-to-MSN synapses have lower release probability. However, CV is also influenced by the number of release sites (Kullmann, 1994). To clarify this, we performed the MPFA (see Materials and Methods) to determine the quantal properties of CB1-to-MSN and MSN-to-MSN synapses. MPFA involves evoking 6 consecutive uIPSCs at $20 \mathrm{~Hz}$ between functionally connected pairs, causing each uIPSC to stabilize at a different release probability (Pr) (Figure $1 \mathrm{k}$ and $\mathrm{l}$ ). The mean amplitudes and variance of each Pr condition are then plotted and fitted with a parabolic 
curve (Figure $1 \mathrm{k}$ and $\mathrm{l}$ ) that is then used to calculate the $\mathrm{Pr}$, number of release site $(N)$, and quantal size $(Q)$ (see Materials and Methods). MPFA revealed that MSN-toMSN synapses had a greater $\operatorname{Pr}$ (CB1-to-MSN, $0.54 \pm 0.04$; MSN-to-MSN, $0.83 \pm 0.07$; unpaired two-tailed $t$-test: $t_{37}=3.67, p=0.00$; Figure $1 \mathrm{~m}$ ). However, CB1-to-MSN pairs had a substantially greater $N$ (CB1-to-MSN, $17.93 \pm 1.99$; MSN-to-MSN, $2.51 \pm 0.38$; unpaired two-tailed $t$-test: $t_{37}=3.89, p=0.00$, Figure $1 \mathrm{n}$ ), suggesting intensive synaptic innervation. No difference in $Q$ was identified, suggesting similar postsynaptic properties (CB1-to-MSN, 52.98 \pm 5.35 ; MSN-to-MSN, 80.58 \pm 20.30 ; unpaired two-tailed $t$-test: $t_{37}=1.90, p=0.07$; Figure 1o).

Collectively, these results demonstrate that feedforward inhibition from $\mathrm{CB}^{+}$FSIs exerts stronger inhibition over MSNs than lateral inhibition from MSN collaterals, partially because of more release sites.

\section{CB1 Signaling Selectively Suppresses Inhibitory Input from CB1 \pm FSIs}

MSNs in the NAc do not express CB1, whereas $\mathrm{CB}^{+}$FSIs do (Winters et al, 2012), predicting that eCBs released upon activation of MSNs may selectively modulate inhibitory transmission from $\mathrm{CB}^{+}$FSIs. To determine this, we compared the sensitivity of uIPSCs between CB1-to-MSN synapses vs MSN-to-MSN synapses to the synthetic CB1 agonist WIN 55212-2 $(5 \mu \mathrm{M})$ during paired recordings. Application of WIN 55212-2 depressed the amplitude of CB1-to-MSN uIPSCs (relative to baseline, $0.33 \pm 0.07$ ), whereas MSN-to-MSN uIPSC amplitude was minimally affected (relative to baseline, $0.98 \pm 0.13$ ), indicating $\mathrm{eCB}$ signaling selectively suppresses CB1-to-MSN inhibitory transmission (RM two-way ANOVA, Cell-type $\times$ Time interaction: $\mathrm{F}_{1,13}=22.01, p=0.00$; $\mathrm{CB} 1$-to-MSN baseline $v s \mathrm{CB} 1$ MSN WIN, $p=0.00$; MSN-to-MSN baseline $v s$ MSN-to-MSN WIN, $p=1.00$; CB1-to-MSN WIN vs MSN-to-MSN WIN, $p=0.00$, Bonferroni posttest; Figure $2 \mathrm{a}-\mathrm{e})$. The reduction in uIPSC amplitudes at CB1-to-MSN synapses by WIN 55212-2 was associated with an increase in the PPR (relative to baseline: CB1-to-MSN, $1.23 \pm 0.06$; MSN-to-MSN, $0.89 \pm 0.029 ; \mathrm{RM}$ two-way ANOVA, Cell-type $\times$ Time interaction: $\mathrm{F}_{1,13}=13.52, p=0.00$; $\mathrm{CB} 1$-to-MSN baseline $v s \mathrm{CB} 1$ to-MSN WIN, $p=0.00$; MSN-to-MSN baseline $v s$ MSN-toMSN WIN, $p=0.37$; CB1-to-MSN WIN vs MSN-to-MSN WIN, $p=0.00$, Bonferroni posttest; Figure 2f) and CV (relative to baseline: CB1-to-MSN, 1.99 \pm 0.22 ; MSN-toMSN, $0.89 \pm 0.12$; RM two-way ANOVA, cell-type $\times$ time interaction: $\mathrm{F}_{1,13}=11.07, p<0.01$; CB1-to-MSN baseline $v s$ CB1-MSN WIN, $p=0.00$; MSN-to-MSN baseline $v s$ MSN-toMSN WIN, $p=1.00$; CB1-to-MSN WIN vs MSN-to-MSN WIN, $p=0.00$, Bonferroni posttest; Figure $2 \mathrm{~g}$ ), consistent with the typical CB1-mediated inhibition of presynaptic release observed throughout the brain (Castillo et al, 2012).

Tonic eCB signaling, which can be triggered by spontaneous release or neuronal activities, modulates basal synaptic transmission in the hippocampus, cerebellum, and dorsal striatum (Adermark and Lovinger, 2009; Kreitzer and Regehr, 2001; Lee et al, 2015). We examined this possibility at CB1-to-MSN synapses by testing the sensitivity of these synapses to the CB1-selective inverse agonist AM251 $(2 \mu \mathrm{M})$ at a dose that effectively prevents $\mathrm{CB} 1$ activation (Chevaleyre and Castillo, 2003; Kreitzer and Malenka, 2005; Lee et al, 2015; Yin and Lovinger, 2006). Application of AM251 slightly increased the amplitude of uIPSCs at CB1-to-MSN synapses (relative to baseline during AM251, 1.16 \pm 0.06 ), indicating tonic eCB-mediated suppression of CB1-to-MSN transmission (paired two-tailed $t$-test: $t_{9}=2.62, p=0.03$; Supplementary Figure S1a-c). This increase in amplitude was associated with a decrease in PPR (relative to baseline: $0.88 \pm 0.05 ;$ paired two-tailed $t$-test: $t_{9}=2.50, \quad p=0.34$; Supplementary Figure S1d), but no change in CV (relative to baseline: $0.95 \pm 0.05$; paired two-tailed $t$-test: $t_{9}=1.01$, $p=0.34$; Supplementary Figure S1e).

\section{Comparison of Excitatory Inputs with CB1 \pm FSIs and MSNs}

Excitatory inputs are likely the primary driving force for $\mathrm{CB}^{+}$FSI-mediated feedforward inhibition of NAc MSNs. We simultaneously recorded $\mathrm{CB} 1^{+} \mathrm{FSI}$ and $\mathrm{MSN}$ in the NAc, and measured EPSCs evoked by the same electrical stimulation of presynaptic fibers (Figure 3a). Stimulation consistently evoked EPSCs with much larger amplitudes in $\mathrm{CB}^{+}$FSIs $(203.20 \pm 16.97 \mathrm{pA})$ compared with EPSCs in MSNs $(60.33 \pm 8.27 \mathrm{pA})$ for all pairs recorded (paired twotailed $t$-test: $t_{15}=7.38, p=0.00$; Figure $3 \mathrm{~b}$ and $\mathrm{c}$ ). The time to the peak amplitude of EPSCs was consistently faster in $\mathrm{CB}^{+}$ FSIs $(1.54 \pm 0.06 \mathrm{~ms})$ than MSNs $(2.78 \pm 0.15 \mathrm{~ms})$ (paired two-tailed $t$-test: $t_{15}=10.05, p=0.00$; Figure $3 b$ and $d$ ). Furthermore, the decay kinetics of evoked EPSCs were also faster in $\mathrm{CB}^{+}$FSIs (CB1, $\tau=2.31 \pm 0.13 \mathrm{~ms}$; MSN, $\tau=4.02 \pm 0.20 \mathrm{~ms}$; paired two-tailed $t$-test: $t_{15}=6.99$, $p=0.00$; Figure $3 \mathrm{~b}$ and e). To determine whether the different magnitudes of evoked EPSCs were because of presynaptic or postsynaptic differences, we assessed PPR and $\mathrm{CV}$. Both $\mathrm{CB}^{+}$FSIs and MSNs had relatively large PPRs $\left(\mathrm{CB}^{+} \mathrm{FSI}, 1.99 \pm 0.10\right.$; MSN, $\left.1.58 \pm 0.15\right)$, suggesting glutamatergic afferents within the NAc have a low probability of presynaptic release relative to inhibitory inputs (Figure 1h). $\mathrm{PPR}$ in MSNs was significantly lower than $\mathrm{CB}^{+}$FSIs, suggesting excitatory inputs to MSNs have a higher release probability (paired two-tailed $t$-test: $t_{15}=2.33, p=0.034$; Figure 3f). In contrast, $\mathrm{MSNs}$ displayed a greater $\mathrm{CV}$ $(0.36 \pm 0.03)$ compared with $\mathrm{CB}^{+}$FSIs $(0.27 \pm 0.01)$ (paired two-tailed $t$-test: $t_{15}=2.981, p<0.01$; Figure $3 \mathrm{~g}$ ). As mentioned above, $\mathrm{CV}$ is also indicative of the number of release sites in addition to release probability, and the greater CV observed here could be because of a fewer number of release sites to MSNs. These presynaptic differences, however, do not fully explain the differences in the EPSC amplitudes at these two synapse types, suggesting additional postsynaptic differences being involved. Collectively, our results suggest that $\mathrm{CB}^{+}$FSIs receive more functional excitatory inputs than MSNs in the NAc.

The results above (Figure 1 ) show that excitation of $\mathrm{CB}^{+}$ FSIs resulted in subsequent inhibition of MSNs. To determine whether excitatory inputs drive disynaptic inhibition of MSNs through $\mathrm{CB}^{+}{ }^{+}$FSIs, a hallmark of feedforward inhibition, we simultaneously recorded an MSN and a $\mathrm{CB}^{+}$ FSI. We recorded the MSN in the voltage-clamp mode and held it at $-45 \mathrm{mV}$ to allow for the detection of IPSCs, while recording $\mathrm{CB}^{+}$FSIs in the current clamp mode to monitor action potential firing. Activation of excitatory inputs evoked 

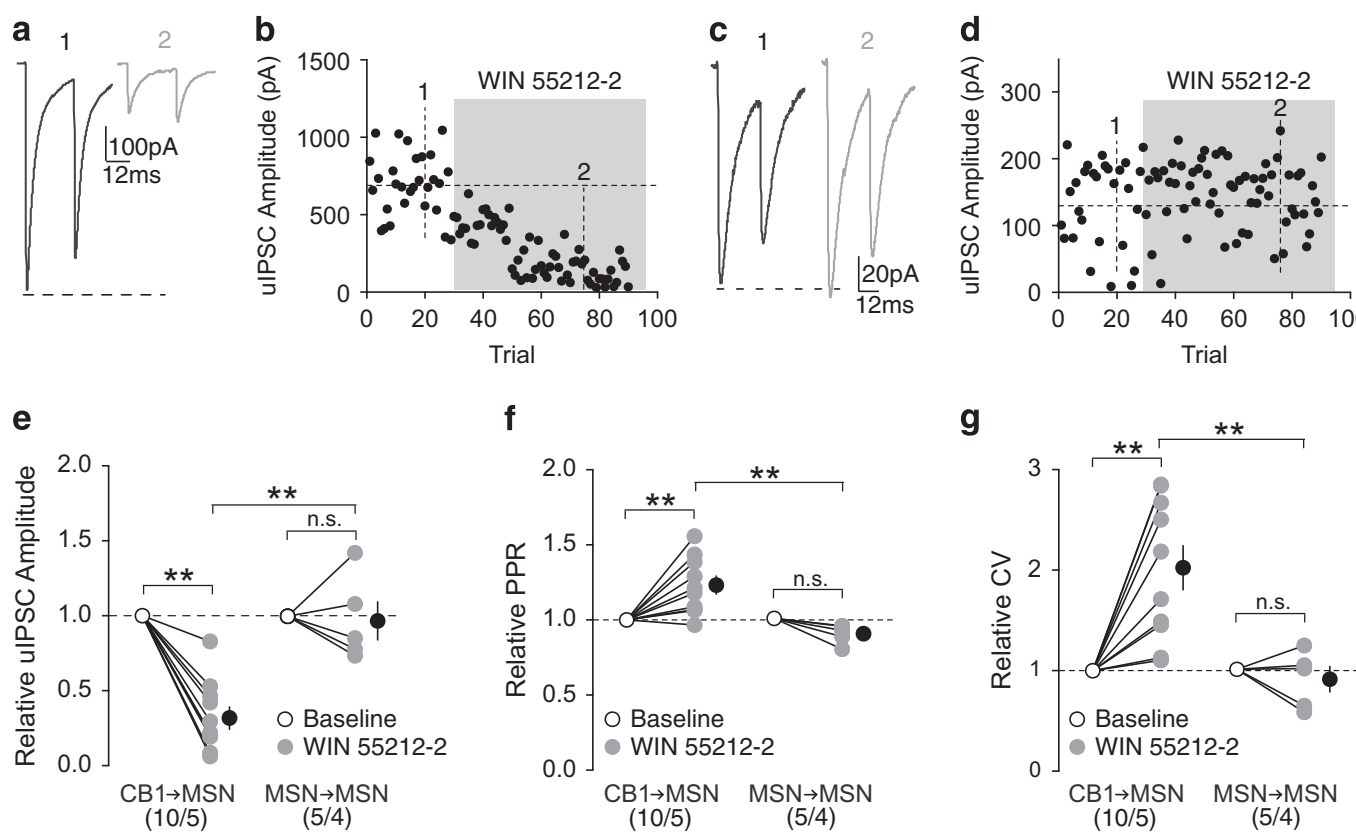

Figure $2 \mathrm{CBI}$ signaling preferentially suppresses inhibitory input from $\left.\mathrm{CB}\right|^{+}$FSIs. (a, b) uIPSC traces (a) and time course of ulPSC amplitude (b) from an example of functionally connected CBI-to-MSN pair before (I) and during (2) perfusion of WIN 552I2-2 (5 $\mu M$ ). (c, d) uIPSC traces (c) and time course of uIPSC amplitude (d) from an example of functionally connected MSN-to-MSN pair before (I) and during (2) perfusion of WIN 552 I 2-2 (5 HM). (e) Summary showing perfusion of WIN 552I 2-2 decreased the amplitude of uIPSCs at CBI-to-MSN synapses, whereas ulPSCs at MSN-to-MSN synapses were insensitive to WIN 55212-2. (f) Summary showing that perfusion of WIN 552I2-2 increased the PPR of ulPSCs at CBI-to-MSN synapses, whereas uIPSCs at MSN-toMSN synapses were unaffected. (g) Summary showing that perfusion of WIN 552I2-2 increased the CV of ulPSC responses at CBI-to-MSN synapses, whereas uIPSCs at MSN-to-MSN synapses were unaffected. $\mathrm{n} / \mathrm{m}$ represents number of cells/number of animals. *** $<0.01$. Error bars represent SEM.

a fast inward current (EPSC) in the MSN, followed by a short-delayed outward current (IPSC), indicative of disynaptic inhibition (Supplementary Figure S2a). When the $\mathrm{CB}^{+}$FSI was switched to voltage-clamp mode and held at $-70 \mathrm{mV}$ to prevent action potential generation, the same excitatory input elicited inward current with increased amplitudes $(2.06 \%$ baseline; paired two-tailed $t$-test: $\left.t_{5}=7.90, p=0.00\right)$, whereas the outward current disappeared $\left(-0.83 \%\right.$ baseline; paired two-tailed $t$-test: $\left.t_{5}=7.56, p=0.00\right)$, demonstrating $\mathrm{CB}^{+}$FSIs as the source of the disynaptic inhibition of MSNs (Supplementary Figure S2a-c).

Given the differences in basal excitatory input to $\mathrm{CB}^{+}$ FSIs and MSNs, we explored possible differences in eCBmediated modulation of excitatory inputs to these two cell types. We assessed the sensitivity of EPSCs to $\mathrm{CB} 1^{+}$FSIs and MSNs to WIN 55212-2 (5 $\mu \mathrm{M})$ during simultaneous recordings of $\mathrm{CB}^{+}$FSIs and MSNs. Application of WIN 55212-2 depressed the EPSC amplitudes in both $\mathrm{CB}^{+}$FSIs and MSNs to a similar degree (relative to baseline: CB1, $0.70 \pm 0.05$; MSN, $0.75 \pm 0.05$; RM two-way ANOVA, time main effect: $\mathrm{F}_{1,30}=55.98, p=0.00$; CB1 baseline vs CB1 WIN, $p=0.00$; MSN baseline $v s$ MSN WIN, $p=0.00$; CB1 WIN vs MSN WIN, $p=0.60$, Bonferroni posttest; Figure $3 \mathrm{~h}-\mathrm{l}$ ). Reduction in EPSC amplitudes was not accompanied by a change in PPR (relative to baseline: CB1, $0.94 \pm 0.03$; MSN, $0.98 \pm 0.07$; RM two-way ANOVA, time main effect: $F_{1,30}=1.03$, $p=0.32$; Figure $3 \mathrm{~m}$ ), whereas only MSNs displayed an increase in CV during perfusion of WIN 55212-2 (relative to baseline: CB1, $1.16 \pm 0.04$; MSN, $1.39 \pm 0.22$; RM two-way ANOVA, time main effect: $\mathrm{F}_{1,30}=5.99, p=0.02$; CB1 baseline $v s$ CB1 WIN, $p=0.64$; MSN baseline $v s$ MSN WIN, $p=0.04$; CB1 WIN vs MSN WIN, $p=0.31$, Bonferroni posttest; Figure $3 \mathrm{n}$ ). The lack of effects on PPR and CV suggest that there is negligible effect on presynaptic release by WIN 55212-2, inconsistent with the predominant presynaptic effects of CB1 (Castillo et al, 2012). However, because of the relatively low release probabilities under basal conditions, a potential reduction in release probability may be difficult to detect with PPR and CV measurements. Nonetheless, our results show that CB1 activation similarly suppresses excitatory inputs to $\mathrm{CB}^{+}$FSIs and MSNs in the NAc. Importantly, CB1 activation does not alter the relative weight of excitatory inputs to $\mathrm{CB}^{+}$FSIs vs MSNs, as there was no change in the $\mathrm{EPSC}_{\mathrm{MSN}} / \mathrm{EPSC}_{\mathrm{CB} 1}$ ratio during WIN 55221-2 application (aCSF, 0.33 \pm 0.05 ; WIN, 0.36 \pm 0.06 ; paired two-tailed $t$-test: $t_{15}=1.13, p=0.28$; Figure 3o).

The above results suggest eCBs are capable of modulating excitatory inputs to $\mathrm{CB}^{+}$FSIs. However, it is unknown whether $\mathrm{CB}^{+}$FSIs are capable of producing and releasing eCBs. One way to determine it is to test for depolarizationinduced suppression of inhibition (DSI) or excitation (DSE). Previously, it was shown that DSI is not induced at CB1-toCB1 inhibitory synapses, despite the presence of CB1 at these synapses (Winters et al, 2012). Here, we tested for DSE at excitatory inputs to $\mathrm{CB} 1^{+}$FSIs. A brief $(10 \mathrm{~s})$ depolarization to $0 \mathrm{mV}$ reliably induced $\mathrm{DSE}$ in $\mathrm{CB}^{+}$FSIs that was prevented by the CB1-selective antagonist AM251 $(2 \mu \mathrm{M})$ (relative to baseline: aCSF, $0.77 \pm 0.05$; AM251, $0.99 \pm 0.04$; RM two-way ANOVA, Time $\times$ aCSF/AM251 interaction: $\mathrm{F}_{1,33}=12.59, p=0.00$; aCSF baseline $v s$ aCSF DSE, $p=0.00$; 
a

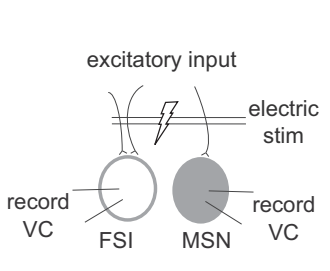

b

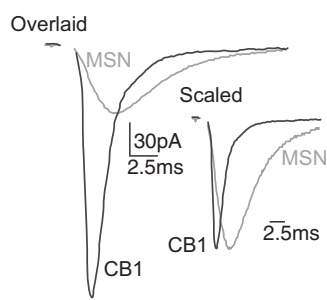

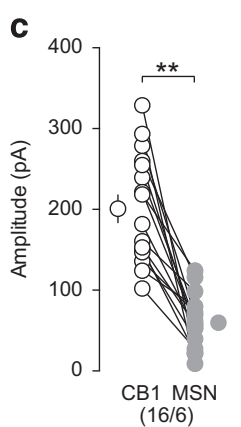

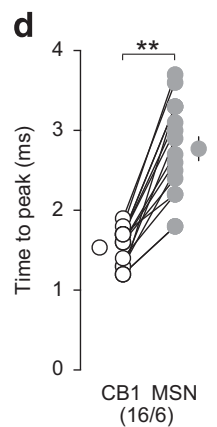

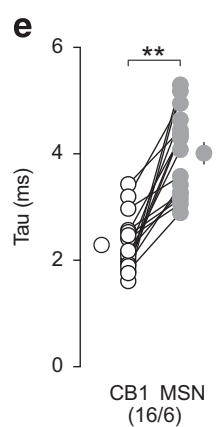

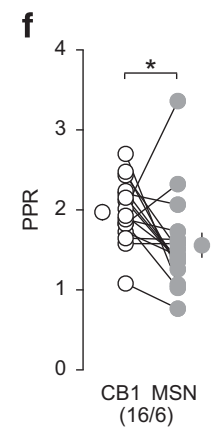

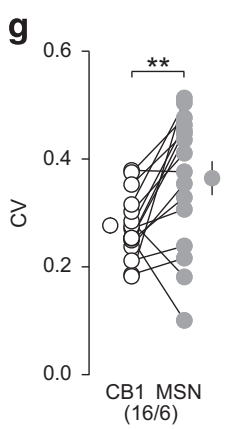

h

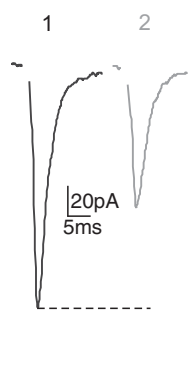

i

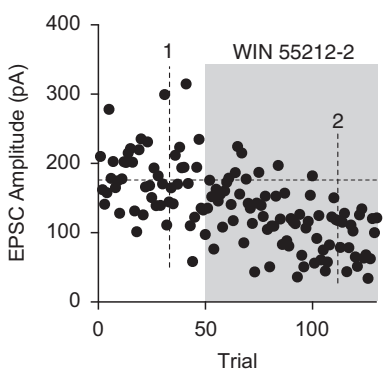

j

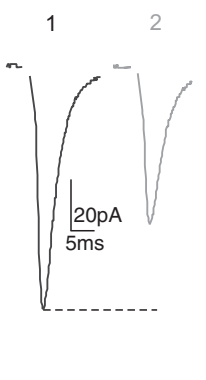

$\mathbf{k}$

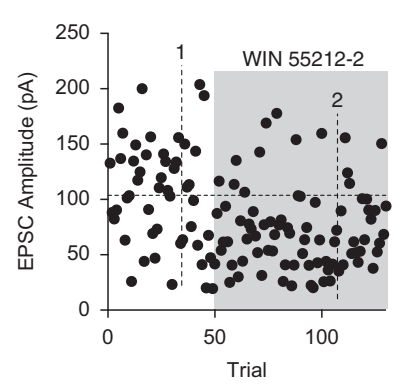

I

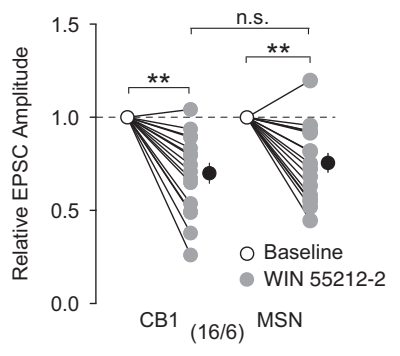

P aCSF

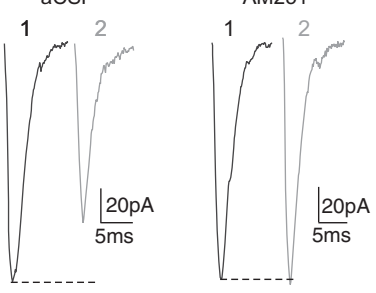

m

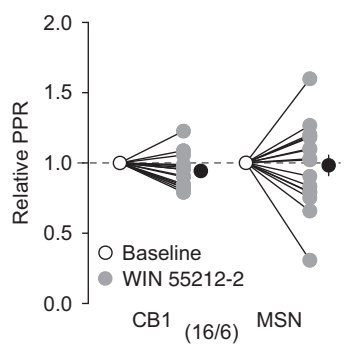

n

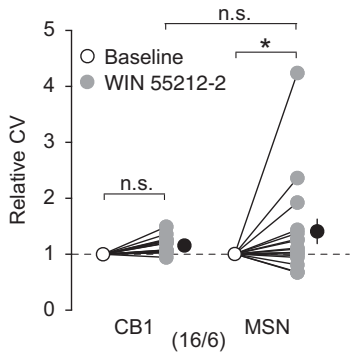

O

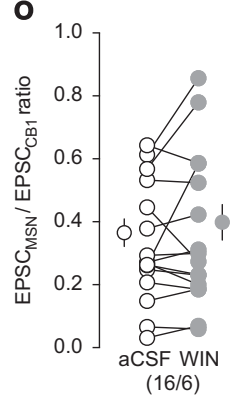

q

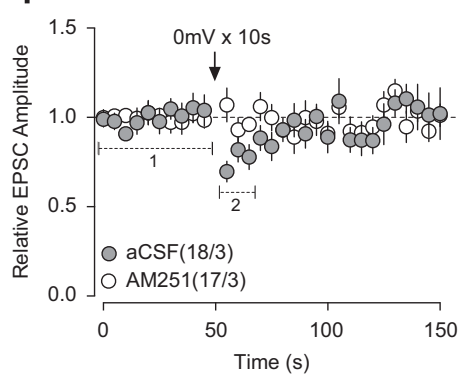

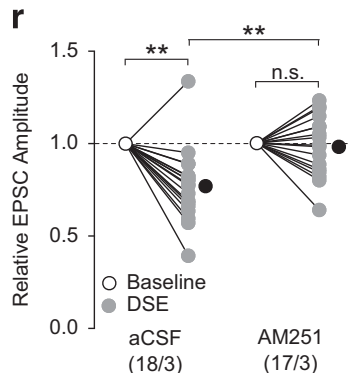

Figure 3 Comparison of excitatory inputs to $\mathrm{CBI}{ }^{+}$FSIs and MSNs and their modulation by CBI. (a) Schematic diagram showing simultaneous dual recordings of a CBI ${ }^{+} \mathrm{FSI}$ and an MSN upon the same electrical stimulation of presynaptic excitatory inputs. (b) Example of traces from evoked EPSCs in the simultaneously recorded $\mathrm{CBI}{ }^{+} \mathrm{FSI}$ (black) and MSN (gray). Overlaid traces (left) highlighting differences in amplitude and scaled traces (right) highlighting differences in activation and decay kinetics. (c) Summary showing the amplitudes of EPSCs were larger in CBI ${ }^{+}$FSIs than MSNs. (d, e) Summary showing EPSCs evoked in $\mathrm{CBI}^{+}$FSIs exhibited a shorter time to peak (d) and decay kinetics (e) than EPSCs in MSNs. (f) Summary showing the PPR of EPSCs evoked in $\mathrm{CBI}^{+}$FSIs was greater than EPSCs in MSNs. (g) Summary showing the CV of EPSCs evoked in MSNs was greater than CBI ${ }^{+} \mathrm{FSIs}_{\text {. }}(\mathrm{h}$, i) EPSCs evoked in an example of CBI ${ }^{+} \mathrm{FSI}(\mathrm{h})$ and the time course of the EPSC amplitudes before (I) and during (2) perfusion of WIN 552 I2-2 (5 HM). (j, k) EPSCs evoked in an example of MSN (j) and the time course of EPSC amplitudes ( $k$ ) before ( 1 ) and during (2) perfusion of WIN 552 I 2-2 (5 HM). Examples shown in (h-k) are from a CBI ${ }^{+} \mathrm{FSI}$ and a MSN recorded simultaneously. (I) Summary showing that perfusion of WIN 552I2-2 decreased amplitude of EPSCS evoked in CBI ${ }^{+}$ FSIs and MSNs to a similar degree. (m) Summary showing that perfusion of WIN 552I2-2 did not affect the PPR of EPSCs evoked in CBI ${ }^{+}$FSIs and MSNs. (n) Summary showing that perfusion of WIN 552I2-2 increased the CV of EPSCs evoked in MSNs but not in CBI ${ }^{+}$FSIs. (o) Summary showing that perfusion of

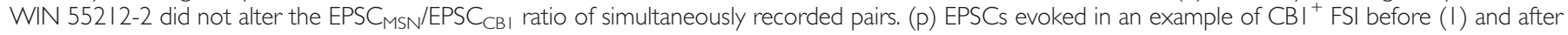

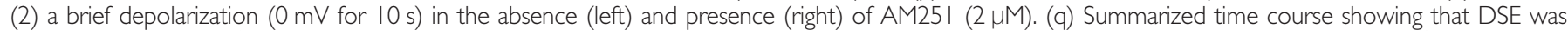
readily induced in $\mathrm{CBI}^{+} \mathrm{FSI}$ and was prevented by $\mathrm{AM} 25 \mathrm{I}(2 \mu \mathrm{M})$. ( $r$ ) Summary showing that DSE decreased the amplitude of EPSCs in CBI ${ }^{+}$FSIs that was blocked by AM25I. n/m represents number of cells/number of animals. $* P<0.05$, $* * *<0.01$. Error bars represent SEM. 
AM251 baseline $v s$ AM251 DSE, $p=1.0$; aCSF DSE $v s$ AM251 DSE, $p=0.00$, Bonferroni posttest; Figure $3 p-r$ ), indicating that the $\mathrm{CB}^{+}{ }^{+}$FSIs are capable of releasing eCBs to modulate excitatory inputs.

Finally, we assessed whether tonic eCB signaling modulates excitatory inputs to $\mathrm{CB1}^{+}$FSIs and MSNs as was demonstrated for CB1-to-MSN synapses. During perfusion of AM251 $(2 \mu \mathrm{M})$, the amplitudes of evoked EPSCs in $\mathrm{CB}^{+}$ FSIs were increased (relative to baseline: $1.21 \pm 0.06$ ), and the EPSC amplitudes in MSNs exhibited a trend toward increase (relative to baseline: $1.14 \pm 0.07 ; \mathrm{RM}$ two-way ANOVA: time main effect, $\mathrm{F}_{1,16}=15.09, p=0.00 ; \mathrm{CB} 1$ baseline $v s \mathrm{CB} 1$ AM251, $p=0.01$; MSN baseline vs MSN WIN, $p=0.08$; CB1 WIN vs MSN WIN, $p=0.64$, Bonferroni posttest; Supplementary Figure $\mathrm{S} 3 \mathrm{a}-\mathrm{c}$ ). The increase in amplitudes was not accompanied by significant changes in PPR (relative to baseline: CB1, $0.93 \pm 0.04 ; \mathrm{MSN}, 0.99 \pm 0.07$; RM two-way ANOVA: time main effect, $F_{1,16}=0.78$, $p=0.39$; Supplementary Figure S3d) nor CV (relative to baseline: CB1, $0.98 \pm 0.09$; MSN, $1.07 \pm 0.15$; RM two-way ANOVA: time main effect, $\mathrm{F}_{1,16}=0.06, p=0.82$; Supplementary Figure S3e). The lack of significant effects may be because the small effects of AM251 were subthreshold for PPR and CV detection. Furthermore, the EPSC $_{\mathrm{MSN}} /$ EPSC $_{\mathrm{CB} 1}$ ratio was not altered by AM251 (aCSF, $0.30 \pm 0.05$; AM251, $0.28 \pm 0.04$; paired two-tailed $t$-test: $t_{8}=1.08$, $p=0.31$; Supplementary Figure S3f), indicating a similar modulation intensity by tonic eCBs at excitatory inputs to $\mathrm{CB}^{+}$FSIs and MSNs.

\section{eCB-Dependent LTD of CB1-to-MSN Synapses}

In addition to short-term plasticity, $\mathrm{eCBs}$ have been implicated in NAc LTD (Robbe et al, 2002). We performed paired recordings to examine unitary $\mathrm{CB}^{+}{ }^{+}$-to-MSN synaptic transmission. After a stable baseline period, we applied LFS ( $2 \mathrm{~Hz}$ for $80 \mathrm{~s}$ ), 3 times with 2 - min intervals through an electrical stimulator placed in the recording area that presumably activated synaptic inputs to both recorded FSIs and MSNs (Figure 4a). This LTD induction protocol was adapted from a similar LFS protocol $(1 \mathrm{~Hz}$ for $80 \mathrm{~s})$ that has been shown to reliably induce eCB-mediated LTD at FSI-toMSN synapses in the dorsal striatum (Mathur et al, 2013). We chose to use the modified $2 \mathrm{~Hz}$ protocol as it produced stronger LTD of UIPSCs in our paired recordings compared with the $1 \mathrm{~Hz}$ protocol (Supplementary Figure S4). MSNs in the NAc transition between a hyperpolarized downstate and a depolarized upstate in vivo (Wilson and Kawaguchi, 1996) that has been shown to gate the induction of eCB-dependent LTD at inhibitory synapses in the dorsal striatum (Mathur et al, 2013). Therefore, we held the postsynaptic MSN at either $\sim-80 \mathrm{mV}$ or $\sim-55 \mathrm{mV}$, mimicking the down- and upstate, respectively, during LFS.

When MSNs were held at $-55 \mathrm{mV}$, LFS to FSI-to-MSN synapses induced pronounced LTD of UIPSCs, whereas the same LFS only marginally depressed uIPSCs at $-80 \mathrm{mV}$ (relative to baseline: $\quad-55 \mathrm{mV}, \quad 0.65 \pm 0.07 ; \quad-80 \mathrm{mV}$, $0.88 \pm 0.02$; RM two-way ANOVA, Time $v s$ Holding interaction: $\mathrm{F}_{1,13}=7.56, p=0.02 ;-55 \mathrm{mV}$ baseline $v s-55 \mathrm{mV}$ LTD, $p=0.00 ;-80 \mathrm{mV}$ baseline $v s-80 \mathrm{mV} \mathrm{LTD,} p=0.16$; $-55 \mathrm{mV}$ LTD $v s-80 \mathrm{mV}$ LTD, $p=0.00$, Bonferroni posttest; Figure $4 \mathrm{~b}-\mathrm{g})$. Decreased uIPSCs from either induction condition was not associated with a change in PPR (\% baseline: $-55 \mathrm{mV}, 1.0 \pm 0.06$; $-80 \mathrm{mV}, 0.99 \pm 0.05$; RM twoway ANOVA, Time main effect: $F_{1,13}=0.03, p=0.87$; Figure $4 \mathrm{~h}$ ), nor a change in the $\mathrm{CV}$ (\%baseline: $-55 \mathrm{mV}$, $1.23 \pm 0.09 ;-80 \mathrm{mV}, 1.0 \pm 0.09$; RM two-way ANOVA, Time main effect: $F_{1,13}=2.532, p=0.14$; Figure $4 \mathrm{i}$ ). These findings suggest that CB1-to-MSN synapses in the NAc are capable of undergoing activity-dependent LTD, but it is sensitive to the functional states of MSNs.

We next sought to determine the mechanisms mediating this LTD. As LFS only induced pronounced LTD at $-55 \mathrm{mV}$, we focused on this condition. Our PPR and CV measurements suggest that this LTD is not expressed presynaptically, arguing against a CB1-dependent mechanism. However, we also failed to detect changes in PPR and CV of evoked EPSCs following $\mathrm{CB} 1$ activation (Figure $3 \mathrm{~m}$ and $\mathrm{n}$ ), and therefore a potential CB1-mediated mechanism cannot be ruled out based solely on PPR and CV measurements. Furthermore, although not statistically significant, there was an $\sim 20 \%$ increase in CV following LFS at $-55 \mathrm{mV}$ (Figure 4i), suggesting subtle presynaptic changes. This is supported by previous reports that $\mathrm{PPR}$ and $\mathrm{CV}$ measurements are not always capable of detecting the presynaptic effects of eCBmediated LTD (Pan et al, 2008). Therefore, we first focused on CB1-based mechanisms. Preventing CB1 activation with AM251 $(2 \mu \mathrm{M})$ reduced the magnitude of LTD induced at CB1-to-MSN synapses (relative to baseline: $0.83 \pm 0.02$; Figure 4j-1), but did not abolish it. The incomplete prevention suggests the involvement of additional mechanisms.

Presynaptic CB1 is not the only target for eCBs within the brain; transient receptor potential vanilloid 1 (TRPV1) channels are postsynaptic targets for certain eCBs, such as anandamide (Castillo et al, 2012). TRPV1 activation has recently been shown to contribute to the induction of eCBmediated LTD at excitatory and inhibitory synapses (Chávez et al, 2010, 2014), and TRPV1-dependent LTD at NAc excitatory synapses (Grueter et al, 2010). We next focused on TRPV1. Inhibition of TRPV1 with capsazepine (CPZ; $10 \mu \mathrm{M})$, a TRPV1-selective antagonist, reduced the magnitude of LTD (relative to baseline: $0.82 \pm 0.09$ ), but was not abolished, suggesting TRPV1 activation also contributes to the induction of LTD at CB1-to-MSN synapses (Figure $4 \mathrm{j}-1$ ). As blocking either $\mathrm{CB} 1$ or TRPV1 activation partially reduced the magnitude of LTD, we next tested whether their concurrent activation is key for the full-magnitude expression of LTD. Coapplication of AM251 $(2 \mu \mathrm{M})$ and CPZ $(10 \mu \mathrm{M})$ completely abolished the induction of LTD (relative to baseline: $\mathrm{CPZ}+\mathrm{AM} 251,1.02 \pm 0.09$; one-way ANOVA: $\mathrm{F}_{3,26}=4.22, p=0.01$; control vs CPZ+AM251, $p=0.01$; all other comparisons, $p>0.05$, Bonferroni posttest; Figure $4 \mathrm{j}-1)$. These results demonstrate that activitydependent eCB signaling, through activation of both $\mathrm{CB} 1$ and TRPV1, induces LTD at CB1-to-MSN synapses.

\section{DISCUSSION}

Our current study characterizes several basic properties of the $\mathrm{CB}^{+}$FSI circuits that mediate feedforward inhibition of NAc MSNs. These results may provide essential knowledge in understanding how the output of the NAc is regulated by 
a
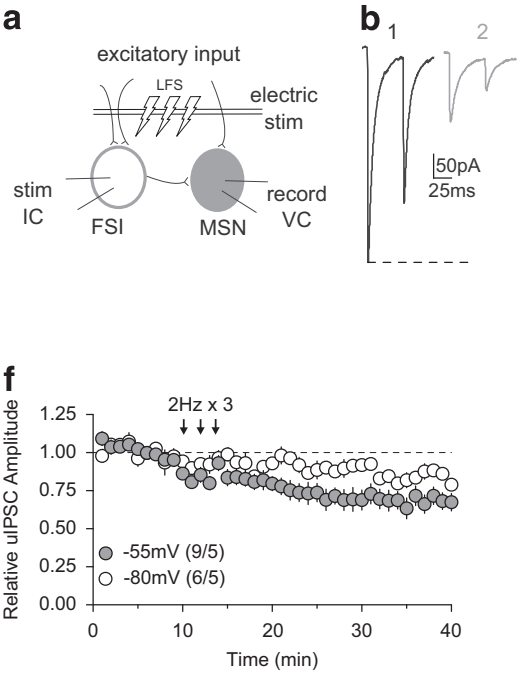

C

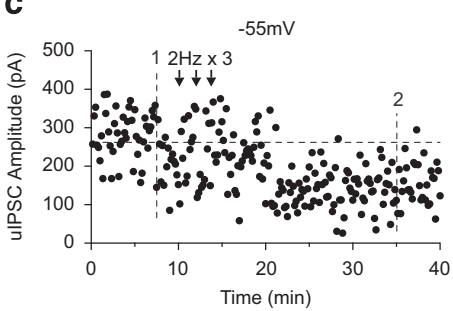

$\mathbf{d}_{1}$

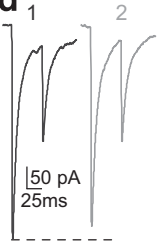

e

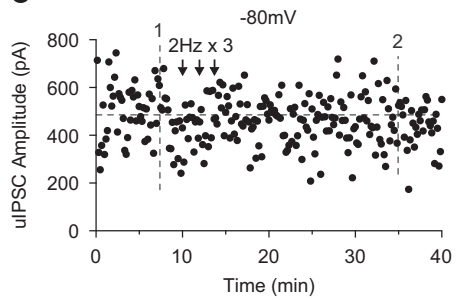

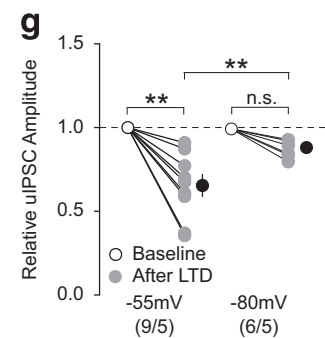

h

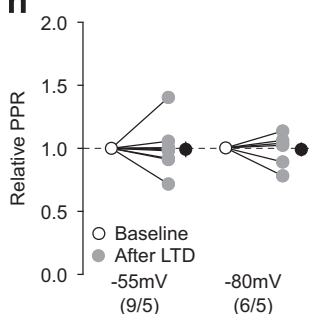

i
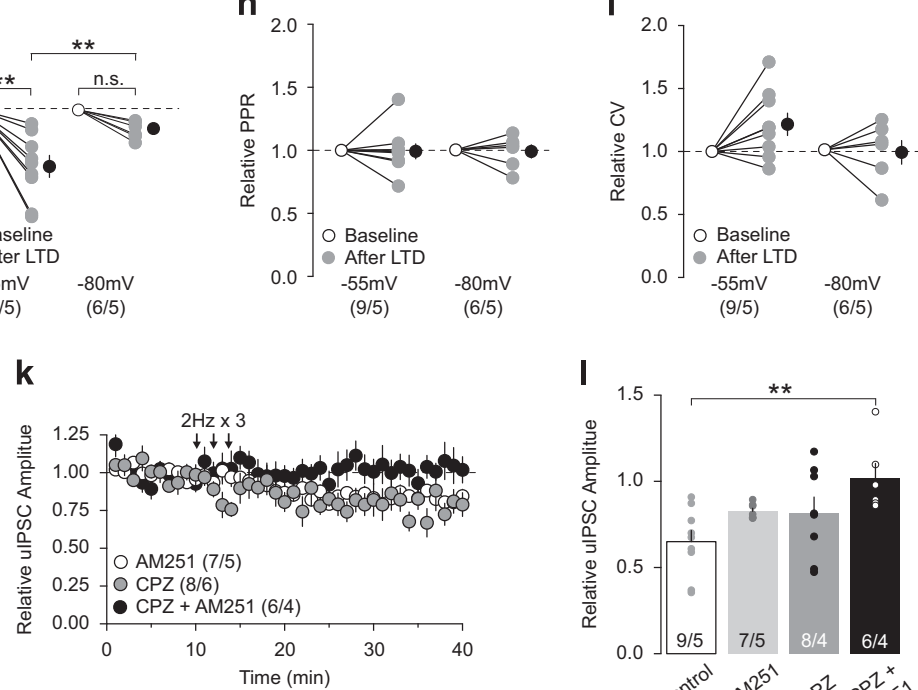

I

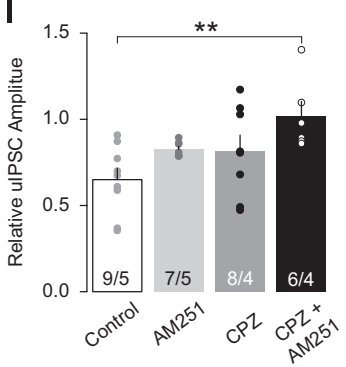

Figure 4 eCB-mediated LTD of CBI-to-MSN inhibitory transmission. (a) Schematic diagram showing paired recordings of a CBI ${ }^{+}$FSI and its connected MSN upon a LFS (2 Hz for $80 \mathrm{~s}$, repeated 3 times) of synaptic inputs. (b, c) uIPSCs (b) and the time course of ulPSC amplitudes (c) from an example of CBI-to-MSN pair before (I) and following (2) LFS when MSNs were held at - $55 \mathrm{mV}$ during delivery of LFS. (d, e) uIPSCs (d) and the time course of ulPSC amplitudes (e) from an example of CBI-to-MSN pair before (I) and following (2) LFS when MSNs were held at - $80 \mathrm{mV}$ during delivery of LFS. ( $f$ ) Summary showing that LFS induced LTD at CBI-to-MSN synapses when MSNs were held at - $55 \mathrm{mV}$, whereas the magnitude of LTD was reduced when MSNs were held at $-80 \mathrm{mV}$ during LFS. (g) Summary showing that the uIPSC amplitudes at CBI-to-MSN synapses were decreased after LFS when MSNs were held at $-55 \mathrm{mV}$ but not $-80 \mathrm{mV}$ during LFS. (h) Summary showing that the PPR of uIPSCs was not significantly affected following LFS. (i) Summary showing that the CV of uIPSCs was not significantly affected following LFS. (j) Example of uIPSC traces from CBI-to-MSN pairs before (I) and following (2) LFS when MSNs were held at $-55 \mathrm{mV}$ during LFS delivery in the presence of AM25I ( $2 \mu \mathrm{M}$; left), CPZ ( I $0 \mu M$; middle), and AM25 I ( $2 \mu M)+C P Z$ (I $0 \mu M)$. (k) Summary showing that LTD was abolished by AM25 I +CPZ, but not by AM25I or CPZ alone. (I) Summary showing that the decrease in uIPSC amplitudes following LFS (control) was partially prevented by AM25I or CPZ alone, but completely prevented by AM25I $+\mathrm{CPZ}$. n/m represents number of cells/number of animals. $* * * 0.01$. Error bars represent SEM.

complex circuit mechanisms under physiological and pathophysiological conditions.

\section{Feedforward and Lateral Inhibitory Control in the NAc}

Local inhibition of NAc MSNs can be mediated by both feedforward and lateral inhibitory circuits, arising from local GABAergic interneurons and MSN axon collaterals, respectively (Tepper et al, 2008). Feedforward and lateral inhibition both influence neural activity patterns, however, in fundamentally different ways. For example, feedforward circuits may act to gate the activation of specific neural ensembles, whereas lateral inhibition may confer winner-take-all properties to competing neural ensembles (Kepecs and Fishell, 2014).

We observed that $\mathrm{CB}^{+}{ }^{+}$FSIs provide a large percentage of neighboring MSNs with powerful inhibitory input, allowing $\mathrm{CB}^{+}$FSIs to exert strong and global influence over the output of nearby MSNs to potentially control ensembles rather than individual MSNs. In contrast to FSIs, MSNs have very sparse connectivity rates and deliver relatively weak inhibition to connected MSNs, thus providing limited influence over a select subset of MSNs. These differential effects are consistent with the circuit roles of FSIs and MSN collaterals in the dorsal striatum (Gittis et al, 2010; Koos and Tepper, 1999; Koos et al, 2004; Taverna et al, 2004). It should also be noted that connectivity rates measured in brain slices can be underestimated as some local connections may be severed during the slice preparation, or the responses between pairs were too small to be distinguished from noises. Regardless, this does not change the conclusion that $\mathrm{CB}^{+}$FSIs serve as the predominating source of local inhibition to MSNs.

uIPSCs at CB1-to-MSN synapses exhibited faster delay and activation kinetics than those at MSN-to-MSN synapses, suggesting that $\mathrm{CB}^{+}$FSIs synapse on proximal or perisomatic locations, whereas MSN collaterals synapse on more distal regions, similar to the anatomical structure in the 
dorsal striatum (Bolam et al, 1983; Kubota and Kawaguchi, 2000; Wilson and Groves, 1980). This differential connectivity confers distinct functional roles, as perisomatic inhibition is positioned to control the spiking output of principle cells (Kepecs and Fishell, 2014), whereas distal inhibition is positioned to gate excitatory inputs (Lovett-Barron et al, 2012; Milstein et al, 2015) and influence synaptic plasticity (Hayama et al, 2013).

Quantal analysis revealed CB1-to-MSN unitary transmission has a larger number of synaptic release sites than MSNto-MSN transmission. Given that central synapses typically possess only one release site (Biró et al, 2005), this greater number of release sites predicts more synapses within CB1to-MSN unitary transmission. On the other hand, MSN-toMSN synapses have a greater release probability of transmitter, although both transmissions exhibit similar quantal sizes. The relatively large number of synapses of the CB1-to-MSN transmission may spread out over more surface area of the MSN to achieve a better overall inhibition, whereas the higher release probability combined with fewer number of synapses may lead to reliable MSN-to-MSN inhibition but confined to specific anatomical sites.

The functionality of feedforward and lateral inhibitory circuits is critically dependent on their activation by excitatory inputs. In response to the same presynaptic stimulation of glutamatergic afferents, $\mathrm{CB}^{+}{ }^{+}$FSIs exhibited much larger amplitude of EPSCs, with faster activation kinetics, compared with adjacent MSNs. As mentioned above, faster activation suggests more proximal synaptic localization. If so, these proximal input to $\mathrm{CB} 1^{+}$FSIs has two perceivable implications: (1) with less dendritic filtering, proximal input may produce larger somatic responses to evoke action potentials; and (2) action potentials fire with a shorter delay. Moreover, with higher membrane resistance, FSIs often exhibit a shorter latency for depolarizationinduced action potential firing compared with MSNs (Winters et al, 2012). These anatomical and biophysical properties allow $\mathrm{CB}^{+}$FSIs to quickly respond to excitatory inputs and fire action potentials to inhibit not only recurrent activation, but also the initial activation of MSNs upon a train of excitatory inputs. In addition, our PPR and CV results suggest that $\mathrm{CB}^{+}$FSIs receive a greater number of excitatory inputs compared with MSNs, whereas inputs to MSNs have a higher probability of release. These notions are consistent with striatal MSNs that receive few and distal innervations from a larger number of individual afferent fibers (Zheng and Wilson, 2002), whereas FSIs receive many and proximal innervations from a smaller number of individual afferents (Ramanathan et al, 2002).

Neuromodulatory circuits comprising cholinergic interneurons (CINs) are also present in the NAc. In contrast to the time-locked control of MSN output mediated by FSIbased feedforward inhibition, CINs have a more modulatory role in regulating MSN function. Cholinergic transmission serves to increase the excitability MSNs through postsynaptic muscarinic receptor signaling (Shen et al, 2005) that may play a permissive role in the induction of long-term plasticity (Calabresi et al, 1999; Shen et al, 2015). In addition, cholinergic transmission regulates the presynaptic release of glutamate and dopamine release (Cachope et al, 2012; Calabresi et al, 1998; Ding et al, 2010; Threlfell et al, 2012). Thus, CINs preferentially regulate the input-to-output processing of MSNs rather than directly influencing MSN activation. In the striatum, CINs may also interact with FSImediated feedforward circuits through monosynaptic connections to FSIs (Koos and Tepper, 2002; Nelson et al, 2014). This potential interaction, however, has yet to be explored in the NAc.

\section{Modulation of Feedforward Inhibition}

Our results suggest that $\mathrm{eCB}$ signaling in the NAc preferentially suppresses $\mathrm{CB}^{+}$FSI-mediated feedforward inhibition, leaving lateral inhibition intact. Specifically, CB1 activation presynaptically depressed inhibitory transmission at CB1-to-MSN synapses. In contrast, inhibitory transmission at MSN-to-MSN synapses was insensitive to CB1 activation, consistent with the selective expression of CB1 in FSIs within the NAc (Winters et al, 2012). This preferential suppression of $\mathrm{CB}^{+}$FSI transmission is in contrast to the dorsal striatum, where CB1 activation has been reported to suppress FSI-to-MSN and MSN-to-MSN inhibitory transmission (Freiman et al, 2006). eCBs also modulate excitatory transmission to MSNs in the NAc (Robbe et al, 2001), and we found that excitatory inputs to $\mathrm{CB}^{+}$FSIs and MSNs were similarly suppressed by CB1 activation. Furthermore, we observed that $\mathrm{CB}^{+}{ }^{+}$FSIs were capable of releasing eCBs to excitatory synapses, and this is in contrast to select inhibitory synapses onto $\mathrm{CB}^{+}$FSIs (Winters et al, 2012). Moreover, our results show that eCB signaling suppresses the glutamatergic inputs to FSIs and MSNs with similar magnitudes, and this eCB-mediated suppression of excitatory transmission is substantially less than the suppression of CB1-to-MSN inhibitory transmission. Collectively, the net effect of CB1 activation in the NAc may result in the preferential suppression of feedforward inhibition arising from $\mathrm{CB} 1^{+}$FSIs.

eCB signaling triggers long-term adaptations in synaptic transmission, most commonly LTD (Castillo et al, 2012). eCB-mediated LTD has previously been demonstrated at FSI-to-MSN synapses in the dorsal striatum, where it has a lower induction threshold compared with LTD at excitatory synapses (Adermark and Lovinger, 2009; Mathur et al, 2013). Our current results demonstrate that activity-dependent eCB signaling triggers LTD of FSI-to-MSN transmission in the NAc. This form of eCB-LTD is dependent on both presynaptic activation of $\mathrm{CB} 1$ and postsynaptic activation of TRPV1 for a full induction. A similar CB1- and TRPV1dependent mechanism has also been demonstrated to mediate eCB-dependent LTD at excitatory synapses in the NAc, triggering a reduction in presynaptic release and endocytosis of postsynaptic receptors (Grueter et al, 2010). However, these conclusions should be taken with caution, as forebrain expression of TRPV1 has recently been called into question with novel genetic labeling methods (Cavanaugh et al, 2011), although this study may not detect unidentified alternative splice variants. Furthermore, the TRPV1 antagonist, capsazepine, used in the current study has off-target effects, including inhibition of voltage-gated calcium channels (Docherty et al, 1997) that are critically involved in synaptic plasticity.

Collectively, as MSNs fluctuate between depolarized upstates and hyperpolarized downstates in vivo (Wilson and Kawaguchi, 1996), the voltage sensitivity identified here 
may gate the induction of LTD at CB1-to-MSN synapses when MSNs are in the upstate and likely be active, potentially favoring subsequent activation through disinhibition of $\mathrm{CB}^{+}$FSIs.

\section{Concluding Remarks}

Our study provides an initial characterization of feedforward and lateral inhibition in the NAc arising from $\mathrm{CB}^{+}$FSIs and MSN collaterals, respectively. In addition, we identify disinhibition of MSNs from feedforward inhibition as a major target for eCB signaling in the NAc. Although our findings provide a framework for conceptualizing how the inhibitory microcircuits shapes the NAc output, future studies are needed to fully understand how the circuits behave when all components are left intact and their role in regulating ongoing behavior. A deeper understanding of the NAc microcircuits in motivated behaviors may open new avenues for the development of novel therapeutic strategies for psychiatric and psychological disorders.

\section{FUNDING AND DISCLOSURE}

The authors declare no conflict of interest.

\section{ACKNOWLEDGMENTS}

We thank Kevin Tang for technical assistance. This work was supported by the NIH NIDA grants DA23206 (to YD), DA34856 (to YD), DA40620 (to YD), and NINDS grant T32NS007433 (to WJW).

\section{REFERENCES}

Adermark L, Lovinger DM (2009). Frequency-dependent inversion of net striatal output by endocannabinoid-dependent plasticity at different synaptic inputs. J Neurosci 29: 1375-1380.

Biró AA, Holderith NB, Nusser Z (2005). Quantal size is independent of the release probability at hippocampal excitatory synapses. J Neurosci 25: 223-232.

Bolam JP, Somogyi P, Takagi H, Fodor I, Smith AD (1983). Localization of substance P-like immunoreactivity in neurons and nerve terminals in the neostriatum of the rat: a correlated light and electron microscopic study. J Neurocytol 12: 325-344.

Brog JS, Salyapongse A, Deutch AY, Zahm DS (1993). The patterns of afferent innervation of the core and shell in the "accumbens" part of the rat ventral striatum: immunohistochemical detection of retrogradely transported fluoro-gold. J Comp Neurol 338: 255-278.

Cachope R, Mateo Y, Mathur BN, Irving J, Wang H-L, Morales M et al (2012). Selective activation of cholinergic interneurons enhances accumbal phasic dopamine release: setting the tone for reward processing. Cell Rep 2: 33-41.

Calabresi P, Centonze D, Gubellini P, Bernardi G (1999). Activation of M1-like muscarinic receptors is required for the induction of corticostriatal LTP. Neuropharmacology 38: 323-326.

Calabresi P, Centonze D, Gubellini P, Pisani A, Bernardi G (1998). Blockade of M2-like muscarinic receptors enhances long-term potentiation at corticostriatal synapses. Eur $J$ Neurosci 10: 3020-3023.

Castillo PE, Younts TJ, Chávez AE, Hashimotodani Y (2012). Endocannabinoid signaling and synaptic function. Neuron 76: $70-81$.
Cavanaugh DJ, Chesler AT, Jackson AC, Sigal YM, Yamanaka H, Grant R et al (2011). Trpv1 reporter mice reveal highly restricted brain distribution and functional expression in arteriolar smooth muscle cells. J Neurosci 31: 5067-5077.

Chávez AE, Chiu CQ, Castillo PE (2010). TRPV1 activation by endogenous anandamide triggers postsynaptic long-term depression in dentate gyrus. Nat Neurosci 13: 1511-1518.

Chávez AE, Hernández VM, Rodenas-Ruano A, Chan CS, Castillo PE (2014). Compartment-specific modulation of GABAergic synaptic transmission by TRPV1 channels in the dentate gyrus. J Neurosci 34: 16621-16629.

Chevaleyre V, Castillo PE (2003). Heterosynaptic LTD of hippocampal GABAergic synapses: a novel role of endocannabinoids in regulating excitability. Neuron 38: 461-472.

Ding JB, Guzmán JN, Peterson JD, Goldberg JA, Surmeier DJ (2010). Thalamic gating of corticostriatal signaling by cholinergic interneurons. Neuron 67: 294-307.

Docherty RJ, Yeats JC, Piper AS (1997). Capsazepine block of voltage-activated calcium channels in adult rat dorsal root ganglion neurones in culture. Br J Pharmacol 121: 1461-1467.

Freiman I, Anton A, Monyer H, Urbanski MJ, Szabo B (2006). Analysis of the effects of cannabinoids on identified synaptic connections in the caudate-putamen by paired recordings in transgenic mice. J Physiol (Lond) 575: 789-806.

Gittis AH, Nelson AB, Thwin MT, Palop JJ, Kreitzer AC (2010). Distinct roles of GABAergic interneurons in the regulation of striatal output pathways. J Neurosci 30: 2223-2234.

Grueter BA, Brasnjo G, Malenka RC (2010). Postsynaptic TRPV1 triggers cell type-specific long-term depression in the nucleus accumbens. Nat Neurosci 13: 1519-1525.

Hayama T, Noguchi J, Watanabe S, Takahashi N, Hayashi-Takagi A, Ellis-Davies GCR et al (2013). GABA promotes the competitive selection of dendritic spines by controlling local $\mathrm{Ca} 2+$ signaling. Nat Neurosci 16: 1409-1416.

Hyman SE, Malenka RC, Nestler EJ (2006). Neural mechanisms of addiction: the role of reward-related learning and memory. Annu Rev Neurosci 29: 565-598.

Kawaguchi Y (1993). Physiological, morphological, and histochemical characterization of three classes of interneurons in rat neostriatum. J Neurosci 13: 4908-4923.

Kepecs A, Fishell G (2014). Interneuron cell types are fit to function. Nature 505: 318-326.

Koos T, Tepper JM (1999). Inhibitory control of neostriatal projection neurons by GABAergic interneurons. Nat Neurosci 2: 467-472.

Koos T, Tepper JM (2002). Dual cholinergic control of fast-spiking interneurons in the neostriatum. J Neurosci 22: 529-535.

Koos T, Tepper JM, Wilson CJ (2004). Comparison of IPSCs evoked by spiny and fast-spiking neurons in the neostriatum. J Neurosci 24: 7916-7922.

Kreitzer AC, Malenka RC (2005). Dopamine modulation of statedependent endocannabinoid release and long-term depression in the striatum. J Neurosci 25: 10537-10545.

Kreitzer AC, Regehr WG (2001). Retrograde inhibition of presynaptic calcium influx by endogenous cannabinoids at excitatory synapses onto Purkinje cells. Neuron 29: 717-727.

Kubota Y, Kawaguchi Y (2000). Dependence of GABAergic synaptic areas on the interneuron type and target size. J Neurosci 20: $375-386$

Kullmann DM (1994). Amplitude fluctuations of dual-component EPSCs in hippocampal pyramidal cells: implications for longterm potentiation. Neuron 12: 1111-1120.

Kupchik YM, Brown RM, Heinsbroek JA, Lobo MK, Schwartz DJ, Kalivas P (2015). Coding the direct/indirect pathways by D1 and D2 receptors is not valid for accumbens projections. Nat Neurosci 18: $1230-1232$.

Lee S-H, Ledri M, Tóth B, Marchionni I, Henstridge CM, Dudok B et al (2015). Multiple forms of endocannabinoid and 
endovanilloid signaling regulate the tonic control of GABA release.

I Neurosci 35: 10039-10057.

Lovett-Barron M, Turi GF, Kaifosh P, Lee PH, Bolze F, Sun X-H et al (2012). Regulation of neuronal input transformations by tunable dendritic inhibition. Nat Neurosci 15: 423-430, S1-S3.

Luk KC, Sadikot AF (2001). GABA promotes survival but not proliferation of parvalbumin-immunoreactive interneurons in rodent neostriatum: an in vivo study with stereology. Neuroscience 104: 93-103.

Mallet N, Le Moine C, Charpier S, Gonon F (2005). Feedforward inhibition of projection neurons by fast-spiking GABA interneurons in the rat striatum in vivo. J Neurosci 25: 3857-3869.

Mathur BN, Tanahira C, Tamamaki N, Lovinger DM (2013). Voltage drives diverse endocannabinoid signals to mediate striatal microcircuit-specific plasticity. Nat Neurosci 16: 1275-1283.

Meredith GE, Agolia R, Arts MP, Groenewegen HJ, Zahm DS (1992). Morphological differences between projection neurons of the core and shell in the nucleus accumbens of the rat. Neuroscience 50: 149-162.

Milstein AD, Bloss EB, Apostolides PF, Vaidya SP, Dilly GA, Zemelman BV et al (2015). Inhibitory gating of input comparison in the CA1 microcircuit. Neuron 87: 1274-1289.

Mogenson GJ, Jones DL, Yim CY (1980). From motivation to action: functional interface between the limbic system and the motor system. Prog Neurobiol 14: 69-97.

Nelson AB, Bussert TG, Kreitzer AC, Seal RP (2014). Striatal cholinergic neurotransmission requires VGLUT3. J Neurosci 34: 8772-8777.

Nestler EJ, Barrot M, DiLeone RJ, Eisch AJ, Gold SJ, Monteggia LM (2002). Neurobiology of depression. Neuron 34: 13-25.

Pan B, Hillard CJ, Liu Q-S (2008). Endocannabinoid signaling mediates cocaine-induced inhibitory synaptic plasticity in midbrain dopamine neurons. J Neurosci 28: 1385-1397.

Ramanathan S, Hanley JJ, Deniau JM, Bolam JP (2002). Synaptic convergence of motor and somatosensory cortical afferents onto GABAergic interneurons in the rat striatum. J Neurosci 22: 8158-8169.

Robbe D, Alonso G, Duchamp F, Bockaert J, Manzoni OJ (2001). Localization and mechanisms of action of cannabinoid receptors at the glutamatergic synapses of the mouse nucleus accumbens. J Neurosci 21: 109-116.

Robbe D, Kopf M, Remaury A, Bockaert J, Manzoni OJ (2002). Endogenous cannabinoids mediate long-term synaptic depression in the nucleus accumbens. Proc Natl Acad Sci USA 99: 8384-8388.

Shen W, Hamilton SE, Nathanson NM, Surmeier DJ (2005). Cholinergic suppression of KCNQ channel currents enhances excitability of striatal medium spiny neurons. J Neurosci 25: 7449-7458.

Shen W, Plotkin JL, Francardo V, Ko WKD, Xie Z, Li Q et al (2015). M4 muscarinic receptor signaling ameliorates striatal plasticity deficits in models of L-DOPA-induced dyskinesia. Neuron 88: 762-773.

Taverna S, van Dongen YC, Groenewegen HJ, Pennartz CMA (2004). Direct physiological evidence for synaptic connectivity between medium-sized spiny neurons in rat nucleus accumbens in situ. J Neurophysiol 91: 1111-1121.

Tepper JM, Wilson CJ, Koos T (2008). Feedforward and feedback inhibition in neostriatal GABAergic spiny neurons. Brain Res Rev 58: $272-281$.

Threlfell S, Lalic T, Platt NJ, Jennings KA, Deisseroth K, Cragg SJ (2012). Striatal dopamine release is triggered by synchronized activity in cholinergic interneurons. Neuron 75: 58-64.

Wilson CJ, Groves PM (1980). Fine structure and synaptic connections of the common spiny neuron of the rat neostriatum: a study employing intracellular inject of horseradish peroxidase. J Comp Neurol 194: 599-615.

Wilson CJ, Kawaguchi Y (1996). The origins of two-state spontaneous membrane potential fluctuations of neostriatal spiny neurons. J Neurosci 16: 2397-2410.

Winters BD, Krüger JM, Huang X, Gallaher ZR, Ishikawa M, Czaja $\mathrm{K}$ et al (2012). Cannabinoid receptor 1-expressing neurons in the nucleus accumbens. Proc Natl Acad Sci USA 109: E2717-E2725.

Wise RA (1987). The role of reward pathways in the development of drug dependence. Pharmacol Ther 35: 227-263.

Yin HH, Lovinger DM (2006). Frequency-specific and D2 receptor-mediated inhibition of glutamate release by retrograde endocannabinoid signaling. Proc Natl Acad Sci USA 103: 8251-8256.

Zheng T, Wilson CJ (2002). Corticostriatal combinatorics: the implications of corticostriatal axonal arborizations. J Neurophysiol 87: 1007-1017.

Zhou L, Furuta T, Kaneko T (2003). Chemical organization of projection neurons in the rat accumbens nucleus and olfactory tubercle. Neuroscience 120: 783-798.

Supplementary Information accompanies the paper on the Neuropsychopharmacology website (http://www.nature.com/npp) 